\title{
Selective Generation of Individual Raman Stokes Wavelengths using Shaped Optical Pulses
}

\author{
A.Malinowski, K.T.Vu, K.K.Chen, P.Horak, D.J.Richardson \\ Optoelectronics Research Centre, University of Southampton, Southampton, SO17 1BJ, United Kingdom \\ djr@orc.soton.ac.uk
}

\begin{abstract}
We demonstrate efficient frequency translation of high-energy pulsed fiber laser output to individual Raman Stokes orders in a single mode fiber using active pulse shaping.

(C2007 Optical Society of America

OCIS codes: (140.3510) Fiber lasers; (290.5910) Stimulated Raman scattering;
\end{abstract}

\section{Introduction}

Raman scattering in optical fiber [1] provides a convenient and practical mechanism for wavelength conversion, allowing the creation of high power fiber sources at wavelengths which cannot be generated directly by rare-earth doped fiber lasers. Whilst this has been applied with considerable success for CW sources [2,3], allowing the development of fiber Raman lasers, progress in the pulsed regime [4] has received far less attention. This is largely due to the fact that Raman scattering is dependent on the instantaneous power of the pump beam. In general, a pulse propagating in an optical fiber will generate several Stokes wavelengths since parts of the pulse with different instantaneous power undergo different amounts of Raman scattering compromising the quality and efficiency of the frequency conversion process. In principle, as we demonstrate in this paper, this problem can be overcome by working with shaped flat-topped rectangular pulses, since in this case all points across the pulse experience identical Raman gain. As a result it is possible for a pulse to cycle all of its energy through sequential frequency shifts to successive order Stokes components. For specific powers, all of the pulse energy can in theory be shifted to a given Raman order, allowing for very efficient wavelength conversion. We demonstrate the selection of individual Stokes wavelengths with high conversion efficiency by using actively shaped flat-topped pulses generated using a fiber MOPA system. Use of active pulse shaping is important as it allows application of the techniques for fiber laser systems operating at pulse energies well in excess of the saturation energy, thereby permitting $\mathrm{mJ}$ level operation.

\section{Theory}

The basic principles of our scheme can be modelled using a simple rate equation approach describing the transfer of pulse intensity between different frequency components during propagation along the fiber,

$$
\frac{d I(\omega)}{d z}=\int_{0}^{\infty} d \Delta g_{R}(\Delta)\left\{I(\omega+\Delta)[I(\omega)+N(\omega)]-\frac{\omega}{\omega-\Delta}[I(\omega-\Delta)+N(\omega-\Delta)] I(\omega)\right\}
$$

Here, the first term describes the gain at frequency $\omega$ due to stimulated Raman scattering (SRS) from higher frequency components, while the second term describes losses due to SRS into lower frequency components. Spontaneous Raman scattering is included via $N(\omega)$ which is taken to be the intensity of one photon per mode. Figure 1 shows the simulated output spectra for the fiber used in the experiments described below for different input powers. Increasing the pump power leads to effective power transfer into higher Raman-Stokes orders, while lower orders and the pump itself are increasingly depleted, with most of the power concentrated within a single Stokes band for all input powers chosen - at intermediary peak powers energy resides predominantly around just two frequencies separated by the Raman frequency shift. Note that this model neglects all other fiber nonlinearities as well as dispersion. Therefore energy conversion even into high-order Stokes bands is complete, i.e., without any losses apart from the finite quantum efficiency. 


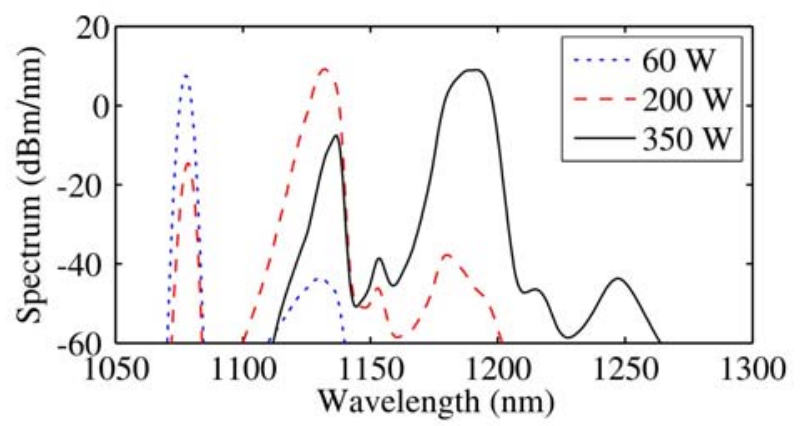

Figure 1. Modelled output spectra for varying input powers for a fiber with $L=41 \mathrm{~m}, A_{\text {eff }}=30 \mu \mathrm{m}^{2}, g_{R}=10^{-13} \mathrm{~m} / \mathrm{W}$.

\section{MOPA system and pulse shaping}

A fully fiberized MOPA system consisting of a directly-modulated semiconductor diode as a seed laser and rareearth doped fiber amplifiers offers great flexibility in varying the pulse parameters, such as pulse duration and peak power. To achieve high pulse energies (mJ regime) and high peak powers (10kW regime) requires gains in excess of $40-50 \mathrm{~dB}$ due to the relatively modest peak powers derivable from the diode seed. At this level of gain and given the relatively modest saturation energies associated with doped fibers, significant pulse reshaping due to depletion of the inversion over the timescale of the pulse can take place within the amplification chain [5]. This has consequences for applications where pulse shape is critical, such as the Raman frequency conversion described here. We have previously demonstrated preshaping of the pulses from the seed laser in order to generate square (and other) output pulses [6]. Here we use our ability to select the desired flat-topped output pulse shape in order to control the Raman frequency conversion. An isolator was used to prevent back reflection to the MOPA.

\section{Stokes generation}

The output of the MOPA was launched into a 41m length of single mode fiber (Corning Hi1060). The maximum total power launched into the fiber was limited to only $1.8 \mathrm{~W}$ when the MOPA was operating at $20 \mathrm{kHz}$, corresponding to a maximum launched pulse energy of $90 \mu \mathrm{J}$. Pulse duration was $100 \mathrm{~ns}$ pulses.
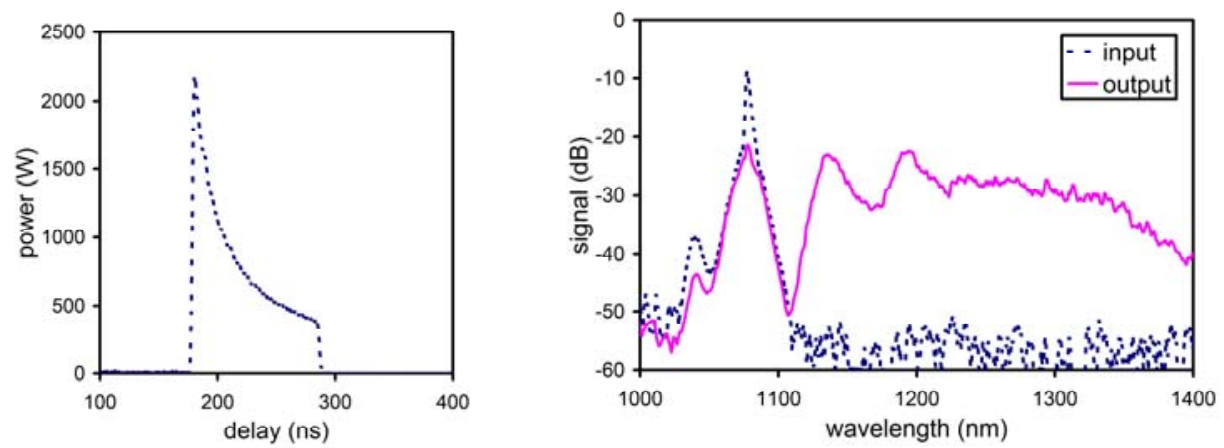

Figure 2. Input pulse shape and spectrum at input (dotted) and output (solid) of single mode fiber without active shaping of seed pulses.

Figure 2 shows the results of Raman generation when no active pulse shaping was applied. In this case the launched pulse of $100 \mathrm{~ns}$ duration has a peak power at the leading edge of around $2 \mathrm{~kW}$, declining to $400 \mathrm{~W}$ in the tail of the pulse. This figure shows efficient Raman conversion with several Stokes orders generated. 

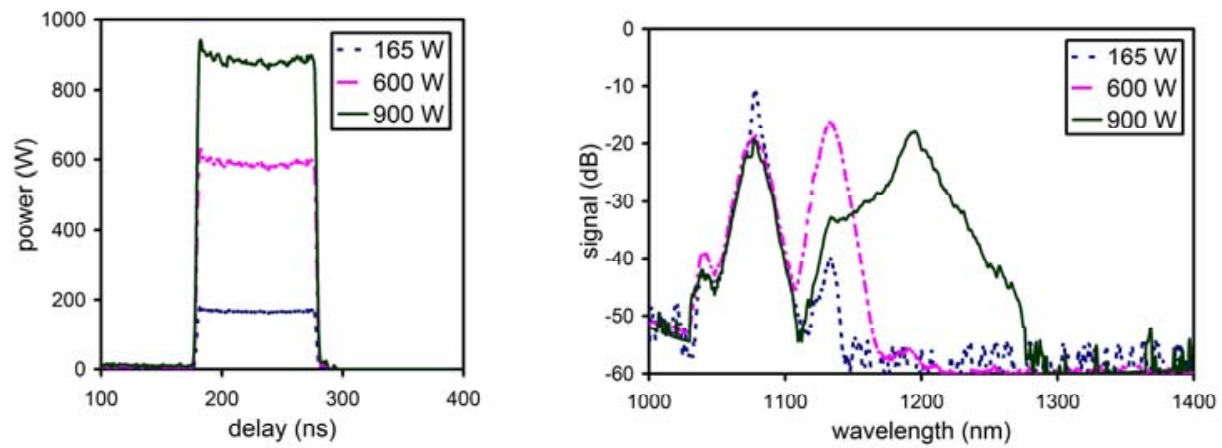

Figure 3. Input pulses and spectra at output from single mode fiber for square pulses created by active shaping of seed pulses at various powers.

Figure 3 shows the spectra obtained when launching square pulses of various power levels. The dotted curve shows the results for an input pulse with a peak power of $165 \mathrm{~W}$. At this power level Stokes generation is insignificant, although the output spectrum is slightly broadened by self-phase modulation (SPM). The dot-dash curve shows the results with a pump peak power of 600W. A significant fraction (75\%) of the pump power has now been transferred to the 1st order Stokes peak. However, no higher order Stokes peak has been generated. In contrast to the numerical simulations we observe a residual pump peak of the order of $25 \%$ which we attribute to pedestals of the flat-topped pulses generated during pulse shaping. The solid curve shows the results for a pump peak power of $900 \mathrm{~W}$. The 1st Stokes order peak has now been suppressed by $15 \mathrm{~dB}$ and most of the pulse energy has been transferred to the 2nd Stokes peak, corresponding to a power of $700 \mathrm{~W}$ in the Stokes pulse. It can be seen that the spectrum progressively broadens due to other nonlinear effects such as SPM and four-wave mixing with increasing degree of wavelength shift, nevertheless distinct spectral peaks are observable. These results confirm the predicted efficient cycling of pulse energy between adjacent Raman orders. Note that the power levels at which particular Stokes orders are excited differ from our predicted values by about a factor of three, which may result from a combination of polarization effects, uncertainty in the precise Raman gain coefficient and the effective area of our fiber, as well as from broad-band amplified spontaneous emission from the pump laser and the pulse pedestal mentioned above. However, the precise origin of this difference is still under investigation. Nevertheless the principle and effectiveness of using shaped pulses is clearly demonstrated.

\section{Conclusions}

In conclusion, we have demonstrated the use of flat-topped pulses for efficient and controllable transfer of pulse energy between sequential Raman orders, showing that almost complete conversion between orders can be achieved by appropriate pulse shape and power control. Importantly, we also show that active pulse shaping can be used to achieve this in fiber MOPA systems operating at pulse energies well in excess of the fiber saturation energy. We consider this to be a most interesting approach to frequency conversion of high power pulsed fiber laser systems.

\section{References}

[1] G. P. Agrawal, Nonlinear Fiber Optics (Academic Press, San Diego, 2001).

[2] J. L. Archambault and S. G. Grubb, J. Lightwave Technol. 15, 1378 (1997).

[3]C.Codemard, P.Dupriez, Y.Jeong, J.K.Sahu, M.Ibsen, J.Nilsson, Optics Letters 2006, 31, 2290-2292, (2006).

[4] P.Dupriez, C.Farrell, M.Ibsen, J.K.Sahu, J.Kim, C.Codemard, Y.Jeong, D.J.Richardson, J.Nilsson, Photonics West, San Jose, CA 21-16 Jan 2006.

[5] L. M. Franz, J. S. Nodvik, ,J. Appl. Phys. 34, 2346-2349 (1963).

[6] K.T.Vu, A.Malinowski, D.J.Richardson, F.Ghiringhelli, L.M.B.Hickey, M.N.Zervas, Opt. Express, 14, 10996-11001, (2006). 